\title{
Opening A New Service Marketing Channel For Medical Products: How Pharmacists Became Immunizers
}

\author{
Catherine Harrington, PharmD, PhD, Nova Southeastern University, USA \\ Holly H. Anderson, PharmD, Nova Southeastern University, USA \\ Daniel J. Vacca RPh, MBA, Nova Southeastern University, USA \\ Nile M. Khanfar, PhD, MBA, Nova Southeastern University, USA
}

\begin{abstract}
Medical product firms often have limited channels of distribution due to the highly regulated environment in which they operate. Creating a new channel for delivery of a health product is very difficult because it requires political action and requires strong arguments that there is unmet need for the product. Determined leadership and action by the intermediaries involved and evidence that the product can be used safely in the new channel are also essential. Pharmacists, vaccine manufacturers, and public health officials were able to open up a new channel for marketing vaccines by persistent effort over the past 15 years. This paper describes this effort and illustrates the benefits for consumers, manufacturers, pharmacists, pharmacy chains, and the public health infrastructure. A series of lessons learned is provided as guideposts for others to use in changing medical product distribution channels.
\end{abstract}

Keywords: Marketing Channels; Pharmacy Services; Medical Products; Pharmacists; Marketing Strategy; Vaccines

\section{INTRODUCTION/BACKGROUND}

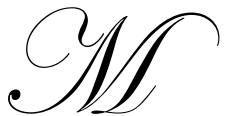

edical product firms often have limited channels of distribution due to the highly regulated environment in which they operate. Generally, they are restricted to small numbers of channels because they are required to sell their products to specific health care facilities licensed by the state to either dispense or administer the product. For example, the manufacturer of a diagnostic test kit might be restricted by law to selling only to centralized laboratories (e.g, Quest Labs), hospitals, or physician offices. Furthermore, insurance coverage practices might further restrict sales by limiting reimbursement to contracted entities (e.g., centralized laboratories), thereby precluding most sales to physician offices. Creating a new channel for delivery of a health product is very difficult because it requires a confluence of three factors; convincing arguments that there is an unmet need for the product or service, determined leadership and action by the intermediaries involved in the new channel, and evidence that the product can be used safely in the new channel. While the investment costs and time involved to create a new channel for a regulated medical product are high, the payoff can be substantial if new sales are obtained by making it easier for consumers to obtain the product. This paper illustrates the creation of a new marketing channel for vaccines by describing how your local community pharmacist can be utilized for the seasonal influenza vaccination.

\section{UNMET NEED TO INCREASE INFLUENZA VACCINATION RATES}

It is wise to identify an unmet need before investing the time and money to create a new marketing channel for a product. The need to increase vaccination rates against influenza in the United State was evident. Annually in the United States (US), influenza accounts for approximately 226,000 hospitalizations. (Thompson 2004) There were 56,000 deaths from influenza and pneumonia in the US in 2006. (CDC, National Vital Statistics 2010) Annual seasonal influenza vaccination is needed to reduce influenza related hospitalizations and deaths, however, many 
people don't receive them for a variety of reasons. Some reasons cited for not receiving vaccinations include people don't like to get shots, it's not convenient to go see the doctor or they don't think they will get sick or they think vaccination will actually make them sick. Because of consumer resistance, there are many challenges to marketing vaccinations. Opening up a new channel could reduce barriers to vaccination by increasing convenience, increasing marketing message exposure via promotional activities, and targeting higher risk groups. High risk groups include the very young children, older adults, minorities and people with co-morbid disease states. Previous public health guidelines recommended that all persons ages 6 to 23 months and over the age of 65 years receive an annual influenza vaccination. (CDC 2004) In 1995, just before the new channel for vaccine administration by pharmacists opened, the percentage of older persons receiving influenza vaccination was 58\%. By 2007, with the new channel opened and pharmacists allowed to vaccinate in 47 states, rates rose to $67 \%$, an increase of 9 points. ("Influenza Vaccination”, 2009)

\section{INFLUENZA IMMUNIZATION SHIFT FROM PHYSICIAN OFFICES TO PHARMACIES}

In the past, a patient being immunized for the seasonal influenza virus associated the event with a visit to their physician's office. In $1999,70 \%$ of flu shots received by persons aged $\geq 18$ years were administered in doctors' offices and other traditional settings.(Singleton, 2005) Recently, vaccination services (for adults, in particular) have been shifting channels away from traditional to nontraditional settings like community pharmacies. This shift can be accounted for primarily by changes in state level legislation that allows pharmacists to immunize. (Traynor, 2009) Lower cost of immunizations in pharmacies (Prosser, 2008), physician acceptance of pharmacists as immunizers (Keely, 2002), and increasing patient awareness of the need for an annual influenza vaccination due to direct marketing by pharmacies (“CVS/pharmacy," 2010).

\section{PHARMACY TAKES UP THE REGULATORY CHALLENGES}

Businesses often face legal and regulatory barriers when attempting to enter a new marketing channel. Community pharmacies involvement in influenza immunization services started in the 1980's when they hosted nurses to administer vaccines during scheduled vaccination clinics. Pharmacists at that time were not legally authorized to administer vaccines. While the use of nurses got around the legal barrier, the real advantage to offering vaccinations services in pharmacies lies in their widespread availability to patients and convenient hours. Having nurses visit pharmacies on a periodic basis for a few hours or days during flu season was not sufficient to exploit the channel. Hiring nurses also increased operating costs.

In 1996, on the advice of a small group of pharmacists and academics (Hogue 2006), pharmacy associations began to take up the charge to obtain authority from state legislators and to train pharmacists for their new role as immunizers. In addition to pharmacy association lobbying and education efforts, vaccine maker (manufacturer) support for the change was critical to the success. All the major vaccine or pharmaceutical manufacturers employ full time lobbyists at the state level to represent company interests. These lobbyists could help the pharmacy associations by providing political support to their efforts to widen access to vaccinations. Manufacturers also provided financial support for training efforts needed to confirm that pharmacists had policies and procedures for safe handling and administration of vaccines. Manufacturer support for pharmacist training continues to this day.

In addition to pharmacist and manufacturer lobbying, the public health community advocated for pharmacists at both the federal and state levels. Public health officials identified a need to have the capacity to vaccinate very large numbers of people quickly in case of emergencies. At the time of the major lobbying effort in 2001, there was a bioterrorism scare from the anthrax attacks. The public health community began to view pharmacies as a key component of their emergency response system. ("The Role of the Pharmacist," 2006) The most recent example of this type of emergency was the H1NI influenza epidemic. ("H1N1 Vaccine," 2009)

Along with the political effort, the pharmacists had to convince insurers that vaccinations in pharmacies are economical. Because of a lower relative overhead in pharmacies, prices for vaccination were set at lower rates. One study found that the mean cost (2004 values) of vaccination was lower in a pharmacy setting (\$US 11.57) than in a scheduled doctor's office visit (\$US 28.67) and concluded using pharmacies to deliver routine influenza vaccination to adults is likely to be cost saving. (Prosser, 2007) 
Within 10 years, nearly all of the legislative barriers had been overcome. In 1994, pharmacists had the authority to administer vaccines in only 12 states. By December 2004, 43 states had authorized pharmacists to administer vaccines. (Hogue, 2006) Currently, all 50 states and the District of Columbia allow pharmacists to administer immunizations. Maine was the last state to legally allow pharmacists to administer immunizations (as of June 2009) and the District of Columbia adopted emergency rules in August 2009 to allow pharmacists to administer adult immunizations via protocol in an emergency. (Traynor, 2009)

\section{PHARMACISTS AS IMMUNIZERS: SAFETY, ACCEPTANCE, AND SATISFACTION}

The American Pharmacists Association, vaccine manufacturers (Sanofi-Aventis, Merck, SmithKline, Wyeth), a pharmacy chain (CVS/Pharmacy), and Boards of Pharmacy (Georgia, Mississippi, National Association of Boards of Pharmacy) worked with the Centers for Disease Control (CDC) and a host of physician and nurse organizations to ensure that pharmacies could provide a safe channel for administering vaccines. A workshop was convened with the National Vaccine Advisory Committee of the CDC that included all the stakeholders. As a result, in 2000, the CDC issued quality standards and program evaluation guidance for adult immunization programs conducted in non-traditional settings including community pharmacies. (Peter, 2000) This agreement among all the stakeholders was essential to convincing policy-makers that vaccinations could be safely delivered in the new pharmacy channel.

Pharmacist as immunizers have gained acceptance from other healthcare providers. Primary care physicians have agreed that pharmacists play a critical role as immunizers for influenza and pneumococcal vaccinations. In a position statement on pharmacist scope of practice in 2002, the American College of PhysiciansAmerican Society of Internal Medicine (ACP-ASIM) supported the role of pharmacist as immunizers in the retail setting. (Keely, 2002)

Patients have accepted pharmacists as immunizers. Community pharmacies provide extended hours of operations and multiple locations that increase access for patients seeking vaccination. Patient convenience is an important factor contributing to the expansion of healthcare services in retail settings and provides another outlet for vaccinating sometimes hard to reach groups such as minorities and the elderly. (Pilisuk, 2010) One study demonstrated the success of this strategy in an elderly group. The study found that individuals aged 65 years and older who lived in states where pharmacists could provide vaccines had were $22 \%$ more likely to receive an influenza vaccination than those who lived in states where pharmacists could not immunize. (Steyer, 2004)

Satisfaction with service and prior exposure by patients has added to the acceptance of pharmacists as immunizers. One study from the Veterans Affairs San Diego Healthcare System stated that patients reported good overall satisfaction with a pharmacist-run immunization clinic in terms of professionalism and access to vaccination (Bounthavong, 2010). In another study, patients recruited in immunizing pharmacies more often reported previous immunization by a pharmacy $(\mathrm{P}<0.001)$, most often for influenza and also reported the immunization was more convenient than a physician office. (Ernst, 2001)

\section{EXPLORING THE NEW CHANNEL}

Pharmacies are benefiting from the new channel through increased revenue as the number of vaccinations administered in pharmacies is growing rapidly. Walgreens reported that a national audit found that pharmacists administered $10 \%$ of all seasonal influenza vaccinations in 2009. Walgreens pharmacists alone administered more than 5.4 million of those vaccinations. ("Retail Pharmacy's Role," 2010) Pharmacy chains are aggressively promoting flu vaccinations by direct email campaigns to their loyalty card members, direct mail advertising to customers, and gift cards sales. The chains have also been aggressively training their pharmacists to administer vaccines. Walgreens has 25,000 pharmacist immunizers ready for 2010 as compared to 16,000 in 2009. (Martin, WSJ, 2010) Pharmacists themselves benefit with greater public recognition of their role as a "hands-on" healthcare provider. In doing so pharmacists are positioning themselves to take on a greater role in delivering primary care as the shortage of non-specialist physicians becomes more acute. (Hoven, 2010) 
Manufacturer's influenza vaccine sales data for the U.S. are difficult to find because vaccines sales may not be reported separately from other drug products. Also, US sales of vaccines and seasonal influenza vaccine sales may not be reported separately. However, distribution data is available (Figure 1) and shows a steady increase over time with a rapid rise since 2005. (Health Industry Distributors Association, 2007-2008)

Figure 1: Influenza Vaccine Production for the U.S. Market in Millions, by Season

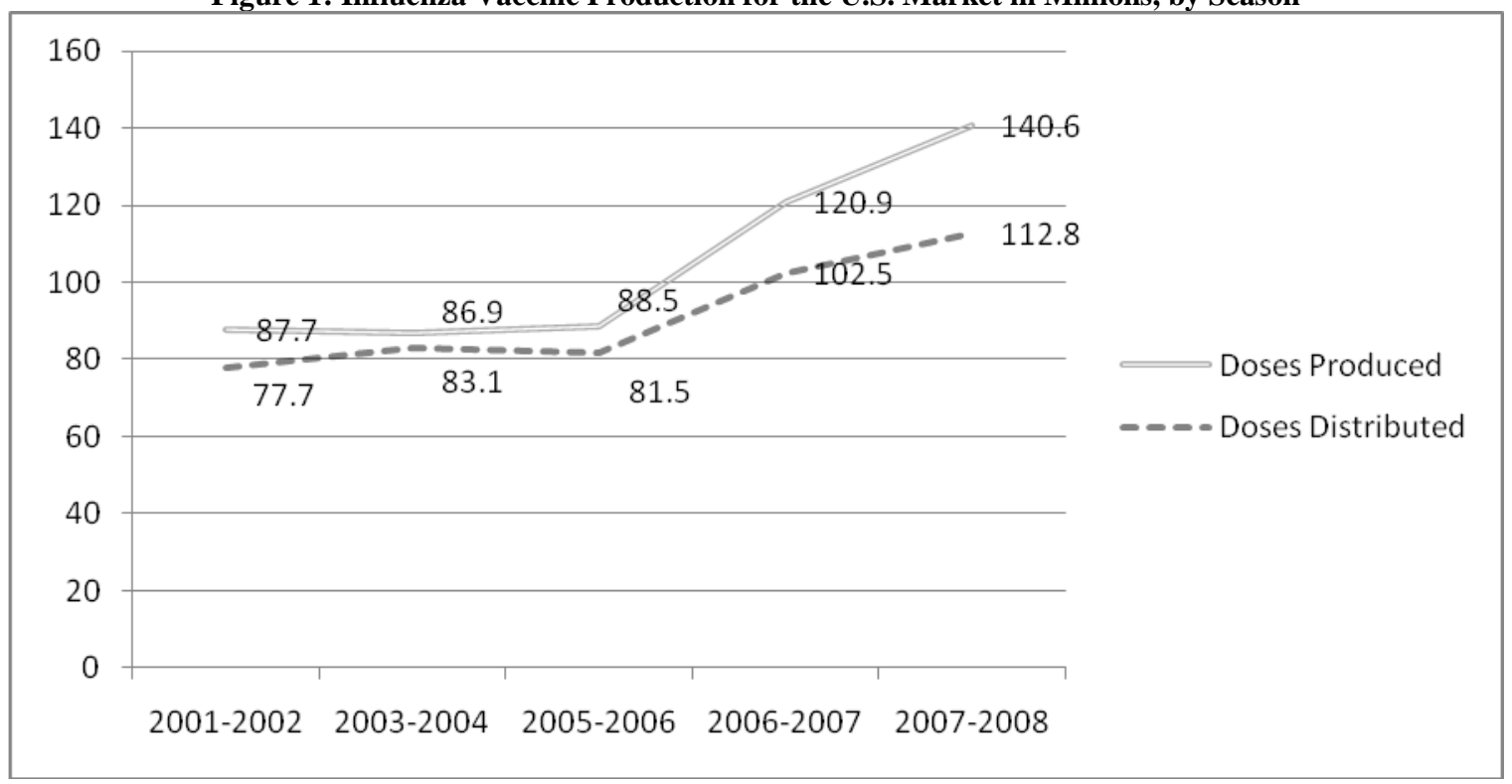

While opening the channel has started to improve influenza vaccination rates across the country, the new pharmacy channel has not been fully exploited. The government is helping out by recommendations to extend annual influenza vaccination to everyone over 6 months of age. (CDC, 2009) This new recommendation for the 2010-11 influenza season will include approximately $99.4 \%$ of the United States population or 308 million people. (US Census 2009) In 2009, only one-third or about 110 million people were vaccinated. There is potential to grow sales by expanding the different types of vaccination offered (e.g., pneumococcal, tetanus, shingles, etc.), expanding the market segments (e.g., children, younger adults), and improving the rate of annual influenza vaccination among minorities.

\section{HOW DOES THE CONSUMER BENEFIT FROM THE NEW CHANNEL?}

A better understanding of the vaccination market is needed to attract more consumers. It is important to examine the attributes of persons currently using the channel to tailor programs and marketing that will continue to attract consumers. What segments are being serviced in the new channel? Does gender, health status, previous immunization history, attitudes, health beliefs, etc. influence the use of a retail pharmacy for seasonal influenza immunization? Attitude can have an enormous impact on the decision of a patient to seek immunization from their pharmacist. Factors influencing a patient's attitude include trust, access, cost, and convenience. There have only been a few published studies (Grabenstein, 2001; Blake, 2003; Grabenstein, 2002 on this subject and all are over seven years old, and since that time there has been broader acceptance of pharmacists as immunizers.

In 2009, the authors (CH and HA) conducted a pilot survey in six stores of a well known national chain pharmacy where pharmacists provided influenza vaccination. The goal was to obtain more current information on the attributes of persons using the pharmacy channel for vaccination. Respondents $(\mathrm{N}=57)$ reflect the demographics of the communities surrounding the pharmacies in that they are highly educated and report good health. Respondents were generally split between males and females. Most had at least one risk factor for influenza, primarily that of being 50 years or older (84\%). Diabetes and respiratory problems in respondents (13\%) 
were higher than state average $(<10 \%)$. Over $25 \%$ of those surveyed did not receive an influenza vaccination the year prior. The likelihood of prior vaccination was linked significantly to older age and having diabetes. Reasons given for not being vaccinated in 2008 were because it was a mild season or they forgot. Of those who did get a vaccination in the prior year, most used a doctor's office to get their immunization.

Of those using the pharmacy in 2009 for vaccination, the majority chose that avenue because it was convenient or personally recommended by someone. Most people chose to get vaccinated in 2009 due fear of illness $(42 \%)$ and/or a doctor's recommendation $(40 \%)$. Respondents liked the times vaccinations were available and that no appointment was needed. They used that particular location because they knew the staff (17\%), the staff told them about the availability of vaccinations (17\%), and/or they trusted the pharmacist (21\%). Their self-rated likelihood of continuing to use the pharmacy for future vaccinations was very high (Figure 2). Comparison of the pilot study data above to the older literature found similarities in age (over 50 years) of respondents. More respondents were female in the older studies as compared to the pilot. Access and convenience of the pharmacy was highly rated in all studies. Cost was less of an issue in the pilot study than in the older studies because Medicare started paying for seasonal influenza vaccinations in 2008.

Figure 2: Likelihood of Continuing Use of Pharmacy for Influenza Vaccination

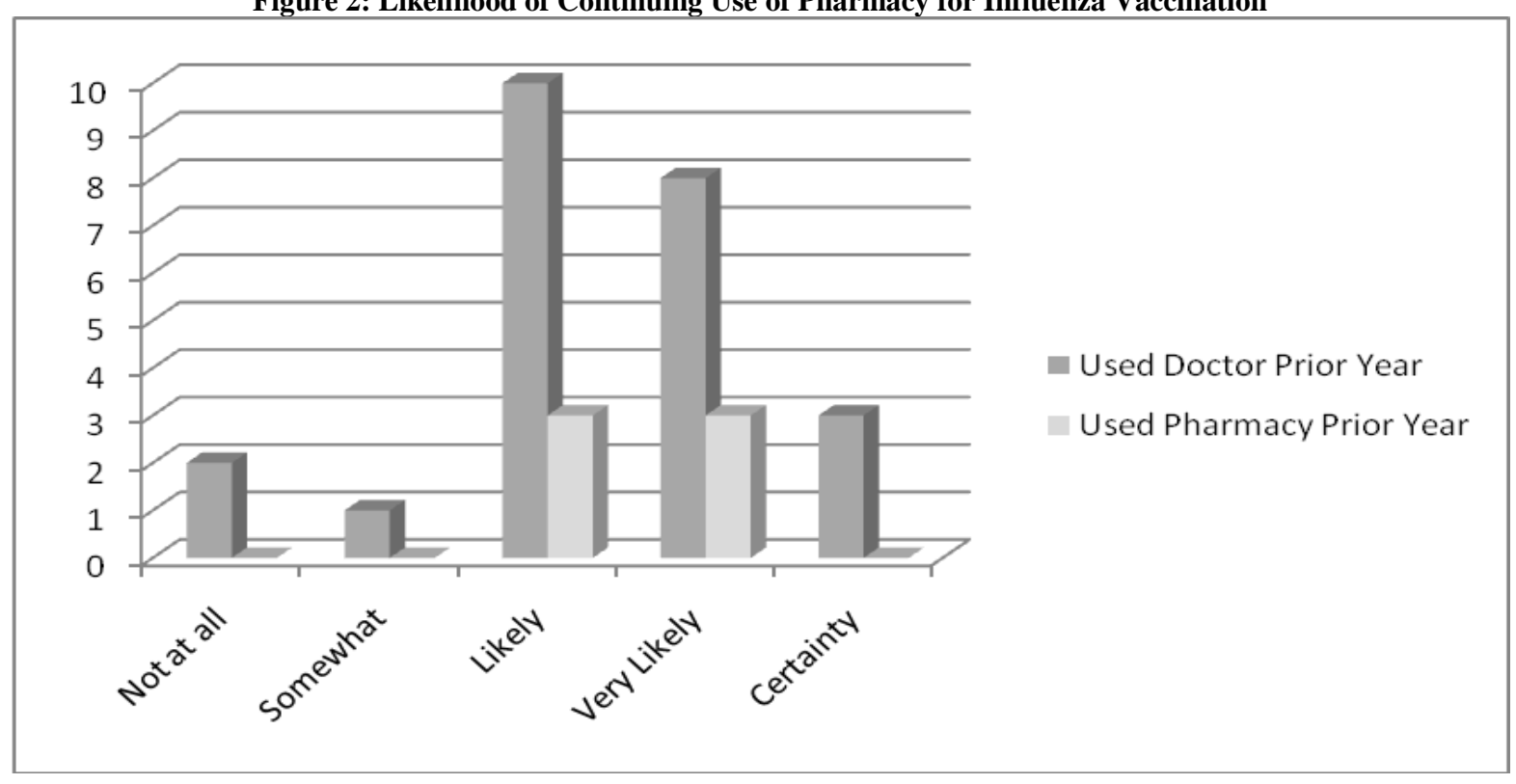

\section{CONCLUSIONS}

Entering new channels for medical product distribution is difficult but possible if teams of health professionals support the effort. Vaccination delivery is complex (e.g., vaccines have to be kept refrigerated, records of use along with lot numbers have to be maintained, etc.) however, pharmacists and their allies were able to open the new channel. Lessons learned from their experience found that it is important to:

1) Define consumer needs

2) Monetize the costs (both monetary and burden of disease) of reduced access

3) Offer a more efficient (less costly and/or more productive) channel

4) Form coalitions with stakeholders, work with mid-level practitioners (e.g., pharmacists, nurse practitioners, physical therapists, etc) demonstrate benefits of using their skills

5) Pro-actively manage channel conflicts. For example, co-opt turf battles with physicians by demonstrating how the new channel will help them care for patients or improve their businesses.

6) Offer continued support of political action 
7) Financially support re-training or new training of new channel participants

8) Once established, exploit the new channel to maximize benefits for everyone.

9) Understand that actual use of the channel by consumers is often fear based and/or physician driven

What new channels await future medical products? Pharmacists are anticipating the day that all types of vaccines will be available to them. Pharmacy benefit managers (PBMs) are in the process of opening up a new channel for genetic testing. They are PBMs in the early stages of commercializing and expanding the availability of genetic testing for in order to enhance individual medication response. (Waters, 2010) Nurse practitioners may want to use portable diagnostic testing devices so that they can do real time monitoring of congestive heart failure patients. Physical therapists should be able to prescribe and administer prescription pain medications (on the spot in rehabilitation clinics) so that more of their patients could withstand the rigors of painful movements. Each of these examples shows that we have underutilized the capabilities of many of our other health professionals. It's time to remove the barriers and improve our health care system in the process.

\section{AUTHOR INFORMATION}

Catherine A. Harrington, PharmD, Ph.D., received both her Doctor of Pharmacy and Doctor of Philosophy in Health Services Organization and Policy from the University of Michigan. She is currently an associate professor at Nova Southeastern University, College of Pharmacy; West Palm Beach, Florida campus. She has held executive positions in pharmacy benefit management and healthcare consultancy businesses. Her research interests include topics in the fields of business, healthcare policy, and drug safety. She has numerous publications in peer-reviewed medical and pharmacy journals.

Holly H. Anderson, PharmD, received her Doctor of Pharmacy degree from Nova Southeastern University in Ft. Lauderdale, Florida. She earned a Bachelor of Science Pharmacy degree from University of Georgia. She is currently an assistant professor and Director of Experiential Education at Nova Southeastern University, College of Pharmacy; Ft. Lauderdale and West Palm Beach, Florida campus. Dr. Anderson holds a consultant pharmacist license in Florida. She has experience working in the Veterans Administration system, in large corporate community pharmacies and in nursing homes. Her research interests include topics in the fields of health and wellness, weight loss, fitness and medication safety.

Daniel J. Vacca, RPh, MBA, received his Bachelor of Science Pharmacy degree from University of Pittsburgh and Masters in Business Administration from Clarion University of Pennsylvania. He is currently an instructor/Clinical Pharmacist at Nova Southeastern University, College of Pharmacy; West Palm Beach, Florida campus. He previously was a Supervisor for a national pharmacy chain and a Manager for a national pharmacy mail service operation. His research interests are in the area of operations, management and finance.

Nile M. Khanfar, Ph.D., MBA, received his Doctor of Philosophy in Pharmacy Administration from the University of Louisiana at Monroe. He is currently an assistant professor at Nova Southeastern University College of Pharmacy; West Palm Beach, Florida campus. Dr. Khanfar has presented several podium and poster presentations at national and international Business and Pharmacy conferences. His research interest includes topics in the fields of marketing, management and healthcare. His work has been published in several peer-reviewed business and pharmacy journals.

\section{REFERENCES}

1. Blake EW, Blair MM, and Couchenour RL. Perceptions of pharmacists as providers of immunizations for adult patients. Pharmacotherapy 2003; 23(2).

2. Bounthavong M, Christopher M, et al. Measuring patient satisfaction in the pharmacy specialty immunization clinic: a pharmacist-run immunization clinic at the Veterans Affairs San Diego Healthcare System. International Journal of Pharmacy Practice. 2010; 18(2):100-07.

3. Centers for Disease Control and Prevention (CDC), Table 1. Deaths, percentage of total deaths, and death rates for the 10 leading causes of death in selected age groups, by race and sex: United States 2006, National Vital Statistics Reports, 2010;58(14):21. 
4. Centers for Disease Control and Prevention (CDC), Prevention and control of influenza: recommendations of the Advisory Committee on Immunization Practices (ACIP) MMWR 2004:53:1-40.

5. Centers for Disease Control and Prevention (CDC), Prevention and control of seasonal influenza with vaccines: recommendations of the Advisory Committee on Immunization Practices (ACIP). MMWR 2009:58:1-520.

6. CVS/pharmacy and MinuteClinic ready for upcoming flu season. CVS/Caremark press release dated 7/26/ 2010. Retrieved from http://info.cvscaremark.com/newsroom/press-releases/cvspharmacy-andminuteclinic-ready-upcoming-flu-season.

7. Ernst M, Bergus G and Sorofman B. Patients' acceptance of traditional and nontraditional immunization providers. JAPhA. 2001; 41(1):53-59.

8. Grabenstein JD, Guess HA. and Hartzema AG. People vaccinated by pharmacists: descriptive epidemiology. J Am Pharm Assoc 2001:41(1):1-9.

9. Grabenstein JD, Guess HA, Hartzema AG, Koch GG, and Konrad TR. Attitudinal factors among adult prescription recipients associated with choice of where to be vaccinated. Journal of Clinical Epidemiology 2002; 55:279-84.

10. H1N1 (swine flu) vaccine at pharmacies. Public Health: Seattle and King County. Retrieved from http://www.kingcounty.gov/healthservices/health/preparedness/pandemicflu/swineflu/pharmacists.aspx.

11. Health Industry Distributors Association, Figure 4: Influenza vaccine production for the U.S. market. 2007-2008 Influenza Vaccine Production \& Distributor Market Brief accessed on 2 Sep 2010 at http://www.preventinfluenza.org/HIDA flubrief07-08.pdf .

12. Hogue MD, Grabenstein JD, Foster SL, and Rothholz MC. Pharmacist involvement with immunizations: a decade of professional advancement. J Am Pharm Assoc 2006; 46:168-182.

13. Hoven AD. Doctor-pharmacist teamwork can apply to many settings. www.Amednews.com posted 16 Aug 2010. Accessed at www.ama-assn.org/amednews/2010/08/16/edca0816.htm.

14. Influenza vaccination among adults 18 years of age and over, by selected characteristics: United States, selected years, 1989-2007, Table 84, Health, United States, 2009.

15. Keely JL, American College of Physicians-American Society of Internal Medicine. Pharmacist scope of practice. Ann Int Med. 2002; 136:79-85.

16. Martin TW. Suntan lotion, flip-flops.....and flu shots. Page B1, The Wall Street Journal 17 August, 2010.

17. Peter G, Meyers M, Marcuse E, Breiman R, Decker M, des Vignes-Kendrick M, Eickhoff T, et.al. Adult immunization programs in nontraditional settings: quality standards and guidance for program evaluation. MMWR 2000:49(RR01):1-13.

18. Pilisuk T, Goad J and Backer H. Vaccination delivery by chain pharmacies in California: Results of a 2007 survey. JAPhA. 2010; 50:2:134-39.

19. Prosser L, O’Brien M, Molinari N, Hohman K, Nichol K, Messonnier M and Lieu T. Non-traditional settings for influenza vaccination of adults: costs and cost effectiveness. Pharmacoeconomics. $2008 ; 26$ (2):163-7.

20. Retail pharmacy's role in flu shot distribution grows 36 percent nationwide. Walgreens press release dated 4/1/2010. Retrieved from http://news.walgreens.com/article display.cfm?article id=5292 .

21. The role of the pharmacist in public health. American Public Health Association 2004, Policy Number: 200614 Revised Policy Date: 11/8/2006.

22. Singleton J, Poel A, Peng-Jun L, Nichol K, Iwane M. Where adults reported receiving influenza vaccination in the United States. Am J Infect Control. 2005; 33:563-70.

23. Steyer T, Ragucci K, Pearson W, Mainous A. The role of pharmacists in the delivery of influenza vaccinations. Vaccine. 2004; 22:1001-06.

24. Thompson WW, Shay DK, Weintraub E, Brammer L, Bridges CB, Cox NJ et al. Influenza-associated hospitalizations in the United States. JAMA 2004; 292:1333-40.

25. Traynor K. With Maine on board, pharmacists in all 50 states can vaccinate. Am J Health Syst Pharm 2009; 66(1):1892-3.

26. U.S. Census Bureau, Population Division. Projections of the population and components of change by net international migration series for the United States: 2010 to 2050 (NP2009-T1). December 16, 2009. Retrieved on 6/2/2010 at www.census.gov/population/www/projections/2009comparisonfiles.html. 
27. Waters R. Drug Managers Medco, CVS giving gene tests to millions in U.S. Bloomberg posted on 11 August 2010 at www.bloomberg.com/news/print/2010-08-11/drug-managers-medco-cvs-giving-gene-teststo-millions-in-u-s.html.

\section{NOTES}

